\title{
ON THE MEAN-VALUE PROPERTY OF HARMONIC FUNCTIONS
}

\author{
MYRON GOLDSTEIN AND WELLINGTON H. OW
}

\begin{abstract}
In this note we show that if the areal mean-value theorem holds for a plane domain (subject to a mild regularity condition) for all integrable harmonic functions, then the domain must be a disk. It is also shown that if a plane domain with finite area has at least two boundary components which are continua then the mean-value property cannot hold for the class of all integrable harmonic functions with single-valued harmonic conjugates.
\end{abstract}

1. In 1962 Epstein $^{1}$ [2] proved the following theorem: "Let $D$ be a simply connected domain of finite area and $t$ a point of $D$ such that, for every function $u$ harmonic in $D$ and integrable over $D$, the meanvalue of $u$ over the area of $D$ equals $u(t)$. Then $D$ is a disk and $t$ its center." In a later paper Epstein and Schiffer extended the above result to domains in Euclidean space $E^{n}$ replacing the simple connectivity hypothesis of the earlier paper by the assumption that the complement of $D$ possess a nonempty interior. Nevertheless, the following theorem strongly suggests that, for plane regions at least, the simple connectedness of $D$ is a necessary condition for the meanvalue property to hold.

TheOREM 1. Let $D$ be a plane domain of finite area having at least two boundary components $\gamma_{1}$ and $\gamma_{2}$ which are continua. Denote by $\mathfrak{H C}$ the class of functions $u$ harmonic in $D$ and integrable over $D$; and by $F \subset \mathfrak{F}$ the subclass consisting of functions possessing single-valued harmonic conjugates in $D$. Then $\mathcal{F}$ (and a fortiori $\mathfrak{H C}$ ) does not satisfy the meanvalue property at any point $t \in D$; that is, it is not the case that there exists a $t \in D$ such that, for all $u \in \mathcal{F}$,

$$
u(t)=\frac{1}{A} \iint_{D} u(z) d x d y,
$$

where $A$ denotes the area of $D$.

Presented to the Society, January 21, 1971; received by the editors September 15, 1970.

AMS 1970 subject classifications. Primary 31A05; Secondary 30A31.

Key words and phrases. Kernel function, mean-value property, principal function, normal operator, boundary component.

1 The authors are grateful to Professor B. Epstein for suggesting this problem to them. 
2. Before proving Theorem 1 we will first establish some preliminary results. Given a point $t$ in a plane region $W$ we denote by $p_{0}$ and $p_{1}$ the principal functions (see, for example, [5]) with respect to the normal operators $L_{0}$ and $L_{1}$ and the given singularity $t$. Here $p_{1}$ is defined with respect to the canonical partition $Q$. The functions $P_{0}$ and $P_{1}$ are defined by

$$
P_{\nu}(z ; t)=p_{\nu}(z ; t)+i p_{\nu}^{*}(z ; t), \quad \nu=0,1,
$$

with

$$
\lim _{\tau \rightarrow 0}\left(P_{\nu}(\tau ; t)-1 / \tau\right)=0
$$

where $\tau$ is a parameter about $t$ and $p_{\nu}^{*}$ is the conjugate harmonic function of $p$.

Let $\widetilde{K}(z ; t)$ be the kernel function for the class $l_{2}(D)$ of square integrable analytic functions $f$ in a domain $D$ which possess singlevalued integrals in $D$. Then we have

Lemma 1. $\tilde{K}(z ; t)=(1 / 2 \pi)\left(P_{0}^{\prime}(z ; t)-P_{1}^{\prime}(z ; t)\right)$.

A proof of this relation may be found in [6]. We also have

Lемма 2. Let $W$ be a plane domain of connectivity $n \geqq 2$, possessing at least two boundary components $\gamma_{1}, \gamma_{2}$, which are disjoint analytic Jordan curves. Then the function $\frac{1}{2}\left(P_{0}-P_{1}\right)$ is not univalent in $W$.

Proof. The function $\frac{1}{2}\left(P_{0}-P_{1}\right)$ is analytic in $W \cup \gamma_{1} \cup \gamma_{2}$. Along $\gamma_{i}, i=1,2$,

$$
d\left(P_{0}+P_{1}\right)-\overline{d\left(P_{0}-P_{1}\right)}=2\left(d p_{1}+i * d p_{0}\right)=0 .
$$

Therefore, on $\gamma_{i}, i=1,2$,

$$
\frac{1}{2}\left(P_{0}-P_{1}\right)=\frac{1}{2} \overline{\left(P_{0}+P_{1}\right)}+\text { const, }
$$

where the constant depends on $\gamma_{i}$. Denote by $\gamma_{i}^{\prime}$ the image of $\gamma_{i}$ under $\frac{1}{2}\left(P_{0}-P_{1}\right)$. Due to the mapping properties of $\frac{1}{2}\left(P_{0}+P_{1}\right)$, we see that if $\frac{1}{2}\left(P_{0}-P_{1}\right)$ is univalent and if the connectivity $n$ is $\geqq 2$, then either $\gamma_{1}{ }^{\prime}$ or $\gamma_{2}{ }^{\prime}$ is not the outer boundary, the region encircled by it being disjoint from the image region. Since this is a contradiction, we conclude that $\frac{1}{2}\left(P_{0}-P_{1}\right)$ is not univalent.

3. Proof of Theorem 1. Assume to the contrary that $\mathfrak{F}$ satisfies the mean-value property at a point $t \in D$. By the Schwarz inequality we observe that for each $f(z) \in l_{2}(D)$, the real and imaginary parts of $f(z)$ are integrable over $D$, and so 


$$
f(t)=\iint_{D} f(z) \cdot A^{-1} d x d y .
$$

On the other hand the equality $f(t)=\iint_{D} f(z) \overline{\tilde{K}(z ; t)} d x d y$ holds for each $f \in l_{2}(D)$. Since $\tilde{K}(z ; t)$ is uniquely determined by its reproducing property we conclude that

$$
\tilde{K}(z ; t)=A^{-1} .
$$

This last relation together with Lemma 1 imply that $\frac{1}{2}\left(P_{0}-P_{1}\right)$ is a linear function of $z$ and hence univalent. If $\gamma_{1}$ and $\gamma_{2}$ are not both disjoint analytic Jordan curves to begin with we can by repeated applications of the Riemann mapping theorem map $D$ conformally onto a domain $D$ with finite area such that $\gamma_{1}$ and $\gamma_{2}$ are mapped onto disjoint analytic Jordan curves. We note that if $\phi$ is the one-to-one conformal map of $D$ onto $\hat{D}$ then $\hat{P}_{0}=P_{0} \circ \phi^{-1}$ and $\hat{P}_{1}=P_{1} \circ \phi^{-1}$ are the corresponding principal functions for $\hat{D}$. Since $\frac{1}{2}\left(P_{0}-P_{1}\right)$ is univalent on $D$ it follows that $\frac{1}{2}\left(\hat{P}_{0}-\hat{P}_{1}\right)$ is univalent on $\hat{D}$. But by Lemma 2 this is a contradiction. Hence $\mathcal{F}$ does not satisfy the meanvalue property at the point $t$ as claimed.

4. We now turn to the theorem of Epstein and Schiffer [3] which is as follows:

Theorem 2. Let $D$ be any domain in Euclidean space $E^{n}$, possessing finite measure, and let the complement of $D$ possess nonempty interior. Suppose that there exits a point $t$ in $D$ such that, for every function $u$ harmonic in $D$ and integrable over $D$, the mean-value of $u$ over $D$ equals $u(t)$. Then $D$ is a sphere with center at $t$.

Epstein and Schiffer remark at the end of their paper that due to the assumption made about the complement of $D$, Theorem 2 leaves open the possibility that there exists a domain $D$ which has finite area, is dense in $E^{n}$, and for which the mean-value property stated in their theorem holds. We now show that for plane regions we can give a sharper formulation of Theorem 2 in that the assumption that the complement of $D$ possess nonempty interior may be replaced by a weaker assumption. Because of this result we are able partially to answer the question posed by Epstein and Schiffer above. Note that the class of functions considered in Theorem 2 corresponds to the class $\mathfrak{H C}$ considered in Theorem 1 when $n=2$.

A plane point set $E$ which is compact is said to belong to the class $N_{B}$ if the unbounded component of the complement of $E$ belongs to the class $O_{A B}$ of Riemann surfaces which possess no nonconstant 
bounded analytic functions (cf. Ahlfors-Beurling [1]). Sets in the class $N_{B}$ are totally disconnected. We now state

TheOREM 3. Let $D$ be a plane domain with finite area having at least one boundary component $\gamma$ which is a continuum. If $\mathcal{H}$ satisfies the mean-value property at a point $t \in D$ then $D$ is a disk.

Proof. Since the mean-value property holds at the point $t$ for $\mathfrak{F}$ it holds for the subclass $\mathfrak{F}$ of functions possessing single-valued harmonic conjugates in $D$. As in the proof of Theorem 1 we see that this implies that $\frac{1}{2}\left(P_{0}-P_{1}\right)$ is univalent. Because of Theorem 2 we may assume without loss of generality that $D$ is dense in the entire plane. When this is so there are two possibilities to consider:

Case 1. Suppose that the complement of $D$ with respect to the extended plane is the union $\gamma \cup A$, where $A$ is the union of an at most countable number of compact sets of class $N_{B}$. Since a set of type $N_{B}$ has 2-dimensional Hausdorff measure zero (cf. Sario-Oikawa [5]), and hence zero area, $A$ has zero area. Also $D$ has finite area by assumption and so $\gamma$ must have infinite 2-dimensional Hausdorff measure. But this is impossible. Hence Case 1 does not in fact occur.

Case 2. Suppose now that the complement of $D$ with respect to the extended plane is the union $\gamma \cup B$, where $B$ is not an at most countable union of sets of type $N_{B}$. By the Riemann mapping theorem the extended plane less $\gamma$ can be mapped conformally onto a disk with $\gamma$ being mapped onto the bounding circle. The image of $B$ under this mapping is a set $\hat{B}$ which is again not an at most countable union of sets of type $N_{B}$. But by Theorems 1,2 of Sakai [4] this implies that $\frac{1}{2}\left(P_{0}-P_{1}\right)$ is not univalent. It follows that Case 2 also does not occur, and this concludes the proof.

\section{REFERENCES}

1. L. V. Ahlfors and A. Beurling, Conformal invariants and function-theoretic nullsets, Acta Math. 83 (1950), 101-129. MR 12, 171.

2. B. Epstein, On the mean-value property of harmonic functions, Proc. Amer. Math. Soc. 13 (1962), 830. MR 25 \#4114.

3. B. Epstein and M. Schiffer, On the mean-value property of harmonic functions, J. Analyse Math. 14 (1965), 109-111. MR 31 \#1388.

4. M. Sakai, On constants in extremal problems of analytic functions. Kōdai Math. Sem. Rep. 21 (1969), 223-225. M R 40 \#341.

5. L. Sario and K. Oikawa, Capacity functions, Die Grundlehren der math. Wissenschaften, Band 149, Springer-Verlag, Berlin and New York, 1969. MR 40 \#7441.

6. M. M. Schiffer, The kernel function of an orthonormal system, Duke Math. J. 13 (1946), 529-540. MR 8, 371.

Arizona State University, Tempe, Arizona 85281

Michigan State University, East Lansing, Michigan 48823 